\title{
ANÁLISE ARQUEOMÉTRICA DE CERÂMICA TUPIGUARANI DA REGIÃO CENTRAL DO ESTADO DO RIO GRANDE DO SUL, BRASIL, USANDO FLUORESCÊNCIA DE RAIOS X POR DISPERSÃO DE ENERGIA (EDXRF)
}

\author{
Irene Akemy Tomiyoshi Bona*, Jorge Eduardo S. Sarkis e Vera Lucia Ribeiro Salvador \\ Centro de Química e Meio Ambiente, Instituto de Pesquisas Energéticas e Nucleares, CP 11048, 05422-970 São Paulo - SP, \\ Brasil \\ André Luis Ramos Soares \\ Departamento de Metodologia do Ensino, Núcleo de Educação Patrimonial, Universidade Federal de Santa Maria, Av. Roraima, \\ s/n, 97000-000 Santa Maria - RS, Brasil \\ Sergio Célio Klamt \\ Departamento de Matemática, Centro de Ensino e Pesquisas Arqueológicas, Universidade de Santa Cruz do Sul, Av. Independência, \\ 2293, 95815-900 Santa Cruz do Sul - RS, Brasil
}

Recebido em 10/1/06; aceito em 28/9/06; publicado na web em 28/5/07

\begin{abstract}
ARCHAEOMETRIC ANALYSIS OF TUPIGUARANI POTTERY FROM THE CENTRAL REGION OF THE RIO GRANDE DO SUL STATE, BRAZIL, BY ENERGY DISPERSIVE X-RAY FLUORESCENCE (EDXRF). Energy dispersive X-ray fluorescence methodology (EDXRF) was used to determine $\mathrm{Al}, \mathrm{Ba}, \mathrm{Ca}, \mathrm{Cr}, \mathrm{Fe}, \mathrm{K}, \mathrm{Mn}, \mathrm{Pb}, \mathrm{Rb}, \mathrm{S}, \mathrm{Si}, \mathrm{Sr}, \mathrm{Ti}, \mathrm{V}, \mathrm{Zn}$ in pottery sherds from seven archaeological sites in the central region of Rio Grande do Sul State, Brazil. The potteries' chemical fingerprints from Ijuí River, Ibicuí Mirim River, Vacacaí Mirim River and Jacuí River were identified. Interactions between sites from the Jacuí River, Vacacaí Mirim River and Ibicui Mirim River could have occurred because some samples from these sites are overlapping in a principal component analysis (PCA) graphic. The pottery provenance could be the same.
\end{abstract}

Keywords: tupiguarani tradition; archaeometry; EDXRF.

\section{INTRODUÇÃO}

Dentre os povos que habitaram o Brasil antes da colonização européia, os Guarani contribuíram de forma marcante na formação do povo brasileiro, principalmente o gaúcho.

Segundo Brochado ${ }^{1}$, sua provável origem vem da região sul dos Rios Madeira - Guaporé na Amazônia. Estavam presentes nos estados do Mato Grosso, Mato Grosso do Sul, oeste do estado de São Paulo, nos estados do Paraná, Santa Catarina, Rio Grande do Sul, além do Uruguai, Paraguai e norte da Argentina.

Eram ágrafos e sua cultura foi transmitida oralmente ou escrita a partir de relatos feitos por exploradores e jesuítas após o contato com os europeus no século XVI e XVII.

Soares ${ }^{2,3}$, a partir de dados históricos e etnográficos, apresenta uma proposta de organização social, na qual os Guarani possuíam um sistema político denominado cacicado. Esses líderes tinham poder de controle sobre o trânsito, as alianças e disputas, além de coordenarem as atividades de guerra, mas não existia um Estado. Não havia imposto ou divisão de trabalho. A produção excedente era utilizada para festas que comemoravam alianças, guerras ou casamentos.

No estado do Rio Grande do Sul os registros arqueológicos e históricos mostram a presença dos Guarani em várias regiões. Em especial no Vale do Rio Jacuí, estudos arqueológicos indicam sua presença desde o A.D. $475^{4}$.

Apesar destes estudos, ainda não foram definidos em termos arqueológicos as relações regulares de troca e intercâmbio de matéria-prima e artefatos com outros grupos. Etnograficamente sabese que os Guarani Mbyá, que atualmente ocupam as regiões Sul e Sudeste do Brasil, vivem em famílias nucleares ou famílias extensas que costumam se visitar ou conviver juntos por algum tempo 5 .

*e-mail: ireneatb@usp.br
O objetivo destas visitas é a "troca de notícias, curas, rezas, remédios, auxílio nas atividades de roça e coleta, cultivares agrícolas e espécies da flora nativa com as mais diferentes utilidades e apoio nos processos de (re)ocupação familiar em uma determinada região" ${ }^{5}$.

Desta forma, dentro da Arqueologia, a importância do estudo de interações entre sítios é a verificação destas redes de parentesco formadas pelas famílias Guarani, que funcionariam como verdadeiros canais de circulação de serviços e permitiriam a distribuição e o remanejamento dos recursos, considerada por Schaden (apud Felipim ${ }^{5}$ ) como princípio fundamental da organização sociocultural Guarani.

A partir de estudos da cultura material em sítios arqueológicos da região, verificou-se que a cerâmica, denominada tupiguarani, era bastante homogênea. Este fato não permitia a identificação do status da pessoa ou da aldeia e nem a hierarquia de produção ${ }^{6}$.

A cerâmica tupiguarani é caracterizada principalmente por ser policrômica, nas cores vermelho e/ou preto sobre um engobo branco ou vermelho. Os potes podem ser sem pintura, simples, corrugados, ungulados ou escovados ${ }^{6-8}$. Ela é idêntica em toda a extensão territorial. As variações são mínimas e estas não permitem determinar antiguidade, estilo ou variação de forma, textura ou dimensões ${ }^{6,9}$.

Entretanto, a vasilha cerâmica, enquanto testemunho, carrega consigo dados referentes ao local de produção, manufatura, utilização e descarte ou abandono. Em todas estas etapas ocorre a incorporação de elementos químicos, que podem ser determinados na análise de elementos químicos majoritários e minoritários.

A arqueometria é um ramo multidisciplinar de pesquisa, que estuda problemas relacionados com a herança cultural. Tem como base a obtenção de informações sobre a origem e história dos achados, análise de material relacionado com a estrutura química e modificações e técnicas de datação ${ }^{10}$. 
Tsolakidou et al. ${ }^{11}$ apresentaram resultados de análises comparativas de cerâmica arqueológica obtidas por análise de ativação de nêutrons (NAA), espectrometria de emissão óptica estimulada por fonte de plasma acoplado indutivelmente (ICP-OES), espectrometria de massas com fonte de plasma acoplado indutivelmente (ICP-MS) e fluorescência de raios X (XRF).

Munita et al. ${ }^{12}$ estudaram depósitos de argila e cerâmica dos Asurini, grupo Tupi, família linguiística Tupi-Guarani do Xingu-Pará. Usaram NAA para caracterização química. Concluíram, a partir da análise multivariada, que a cerâmica era originária de duas fontes de argila.

Appoloni et al. ${ }^{13}$ apresentaram a caracterização química de fragmentos cerâmicos arqueológicos. Utilizaram a técnica de microfluorescência de raios $X(\mu-X R F)$ e verificaram que a composição química do pigmento preto utilizado continha manganês, o vermelho, ferro e o branco, bário.

Felicíssimo et al. ${ }^{14}$ caracterizaram cerâmicas pré-coloniais do Pantanal Sul-Mato-Grossense por espectroscopia de fotoemissão de raios X (XPS) e espectroscopia de massa de íons secundários em modo de tempo de vôo (TOF-SIMS). Classificaram a cerâmica em relação à composição da argila usando análise de agrupamento e componente principal. Verificaram que a coloração preta no interior do fragmento era devido aos procedimentos de secagem e queima.

Em outro trabalho, Felicíssimo et al..$^{15}$, usando as técnicas de emissão de raios $\mathrm{X}$ induzida por partículas (PIXE) e emissão de raios gama induzida por partículas (PIGE), determinaram similaridades e correlações entre as amostras de dois sítios do Pantanal MS e as possíveis fontes de argila.

Adan-Bayewitz ${ }^{16}$ determinou a procedência de potes cerâmicos aplicando o método de XRF, comparando-os com a NAA.

Leung et al. ${ }^{17}$ estudaram a composição química de antigas porcelanas chinesas por EDXRF e concluíram que o uso de dados de composição química juntamente com a análise de componente principal, é sensível à discriminação entre cerâmicas e existem semelhanças entre as cerâmicas de Linjiang e a de Jezhou, de regiões distintas.

Druc et al. ${ }^{18}$ fizeram um estudo petrográfico aliado à análise por EDXRF em amostras de cerâmica da região de Callejón de Huaylas, Peru. Verificaram que por meio da análise de agrupamento é possível a identificação de centros de produção e distribuição de cerâmica pré-histórica.

Este trabalho, portanto, teve como objetivo estudar interações entre grupos que habitaram a região central do Rio Grande do Sul por meio da análise da cerâmica que produziram, utilizando a técnica de EDXRF, associada a métodos estatísticos multivariados, como a análise de componentes principais. Pretendeu-se verificar experimentalmente a existência de interações entre as cerâmicas que levem a informações sobre os grupos que habitaram aquela região.

\section{PARTE EXPERIMENTAL}

As amostras analisadas pertencem à Tradição Tupiguarani e foram encontradas em sítios arqueológicos do Rio Grande do Sul.

Os sítios arqueológicos estudados estão localizados no em torno de alguns rios que podem ser vistos na Figura 1: Rio Ijuí, município de São Luiz Gonzaga ${ }^{19}$; Rio Ibicuí Mirim, município de São Pedro do Sul; Rio Vacacaí Mirim, município de Santa Maria ${ }^{20}$ e Rio Jacuí (Alto e Médio Jacuí), municípios de Ibarama e Agudo.

Em termos de relevo, esses sítios fazem parte de duas regiões. A primeira é a Depressão Central, região de planícies aluviais e coxilhas sedimentares, onduladas com declives, em dezenas de metros. A segunda, o Planalto Médio, região aplanada entre 400 e $800 \mathrm{~m}$ de altitude é sendo que na parte sul ocorre arenito e na parte norte, relevo ondulado.

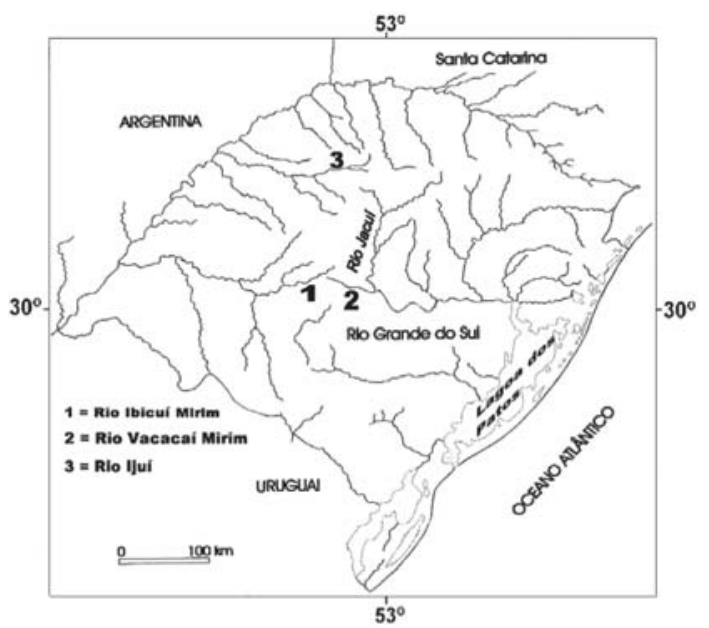

Figura 1. Localização dos sítios arqueológicos estudados: região central do estado do Rio Grande do Sul

As distâncias aproximadas dos sítios estudados em relação ao sítio Wilmoth Röpke, no município de Ibarama no Alto Jacuí são: até o sítio Gentil Menegasse, Ibarama, Alto Jacuí, $5 \mathrm{~km}$; até o sítio Nilton Puntel, Ibarama, Alto Jacuí, 11 km; até o sítio Marsílio Bartz, Agudo, Médio Jacuí, 5 km; até o sítio Cabeceira do Raimundo, Santa Maria, Rio Vacacaí Mirim, 69 km; até o sítio Pedra Grande, São Pedro do Sul, Rio Ibicuí Mirim, 95 km; até o sítio São Lourenço Mártir, São Luiz Gonzaga, Rio Ijuí, 182 km.

Essas distâncias são em linha reta e não têm relação com as distâncias percorridas para se chegar a cada local.

As amostras analisadas são fragmentos cerâmicos pertencentes a vasilhas de diversos formatos e funções, dos tipos simples, pintada, corrugada, corrugada-ungulada e ungulada. As amostras foram enviadas para a realização deste trabalho, após os estudos técnico-tipológicos efetuados pelo Prof. Dr. A. L. R. Soares, Universidade Federal de Santa Maria, RS.

\section{Metodologia}

A técnica usada para determinação da concentração de elementos químicos foi a análise de amostras na forma de pastilhas compactadas, pela técnica de EDXRF.

O equipamento utilizado foi o EDX-900, produzido pela Shimadzu Corporation.

Os parâmetros de operação do espectrômetro foram: tubo de raios $\mathrm{X}$ de Ródio, tensão de $5 \mathrm{KV}$; corrente ajustada automaticamente de no máximo $1 \mathrm{~mA}$; colimador de $10 \mathrm{~mm}$; detector de silício; resfriamento termoelétrico; tempo de medida de $40 \mathrm{~s}$ e 8 varreduras por análise. Utilizou-se suporte para amostra sólida com filme de poliestireno.

O método analítico usado é denominado método dos Parâmetros Fundamentais (PF). Este método permite a obtenção da curva de sensibilidade do equipamento para cada elemento de interesse, quando uma amostra de composição química conhecida é submetida a parâmetros instrumentais bem definidos.

A curva de sensibilidade do equipamento relaciona a intensidade fluorescente teórica calculada e a medida para cada elemento. Desta forma, é possível determinar a composição do material analisado.

A análise dos resultados foi feita usando-se o programa Statistica 6.0, StatSoft Inc. com a aplicação de análises de componentes principais.

A curva analítica foi determinada usando-se os materiais de referência San Joaquim Soil - SRM 2709 e Montana Soil - SRM 2710 e SRM 2711. 
A precisão e a exatidão do método foram verificadas usandose o material de referência de concentração similar aos potes cerâmicos, Brick Clay - SRM 679 e Montana Soil - SRM 2711.

Os elementos determinados foram $\mathrm{Al}, \mathrm{Ba}, \mathrm{Ca}, \mathrm{Cr}, \mathrm{Fe}, \mathrm{K}, \mathrm{Mn}$, $\mathrm{Pb}, \mathrm{Rb}, \mathrm{S}, \mathrm{Si}, \mathrm{Sr}, \mathrm{Ti}, \mathrm{V}$ e $\mathrm{Zn}$

Como a superfície das amostras analisadas não é lisa e pode sofrer alterações elementares ${ }^{21}$, verificou-se a homogeneidade da amostragem e a variação da concentração em função da massa e da pressão de compactação da pastilha. Foi escolhida uma amostra com tratamento de superfície corrugada porque a vasilha com esse tipo de tratamento de superfície poderia ter sido usada para cozer alimentos ${ }^{6} \mathrm{e}$ o fragmento analisado poderia estar contaminado com resíduos dos alimentos e do local em que ficou enterrada. Neste ensaio a massa (2,00 g), a pressão de compactação (10 t) e o tempo de compactação (1 min) foram mantidos constantes.

A dependência com a massa foi verificada por meio da análise de pastilhas com massas de 1,50;2,00 e 5,00 g. Nesse ensaio a pressão (10 t) e o tempo de compactação (1 min) foram mantidos constantes.

Para verificar o efeito da variação de pressão de compactação foram preparadas pastilhas com 10 e 5 t de compactação, sendo constantes a massa (2,00 g) e tempo de compactação (1 min).

Após esses ensaios, as amostras de cerâmica arqueológica foram preparadas usando-se massa constante de 2,00 g, pressão de 10 t e tempo de compactação de $1 \mathrm{~min}$.

\section{Preparação de amostra}

A limpeza da amostra consistiu no polimento das superfícies para retirada de pintura e contaminação da superfície. A lavagem foi feita pelo método ultra-sônico em água desmineralizada; o enxágüe, pelo método ultra-sônico em álcool etílico e posteriormente em acetona. A secagem foi efetuada em estufa a $105^{\circ} \mathrm{C}$ por $18 \mathrm{~h}$.

Após a limpeza da amostra, fez-se a moagem e homogeneização em almofariz de ágata. As amostras foram pesadas e foram obtidas pastilhas de $3 \mathrm{~cm}$ de diâmetro e espessura variada por compactação do pó, usando-se a prensa hidráulica. O almofariz e o pistilo de ágata foram descontaminados com solução de ácido nítrico, $30 \%$. A eficiência da limpeza foi verificada com a análise dos elementos de interesse de uma solução que permaneceu em contado com o almofariz e o pistilo durante $4 \mathrm{~h}$ por HR-ICP-MS.

\section{RESULTADOS E DISCUSSÃO}

Os dados obtidos para os materiais de referência SRM 679 e 2711, usando-se a curva analítica obtida, são apresentados nas Tabelas 1 e 2. Verifica-se que o desvio padrão relativo (DPR) encontrado é $\leq 10 \%$ para os elementos analisados. O coeficiente de variação $(\mathrm{CV})$ é $\leq 10 \%$ para $\mathrm{Al}, \mathrm{Ba}, \mathrm{Ca}, \mathrm{Fe}, \mathrm{K}, \mathrm{Mn}, \mathrm{Pb}, \mathrm{Rb}, \mathrm{Si}, \mathrm{Sr}$, Ti e $\mathrm{V}^{11}$, mas depende do material analisado.
O limite de determinação do método (LDM) é calculado pela Equação 1. Considera-se que na prática, dentro de um contexto analítico, essa forma permite a obtenção de valores mais realísticos que os limites de detecção do método ${ }^{22}$.

$\mathrm{LDM}=2 \cdot \sqrt{\frac{\sum_{i=1}^{N}\left(C_{i}-\bar{C}\right)^{2}}{N-1}}$

onde: $C_{i}$ é a concentração de 10 replicatas analisadas; $\overline{\mathrm{C}}$, a média de 10 replicatas analisadas e $N$, o número de replicatas

Foi determinado o valor do índice $\mathrm{Z}^{23}$ também chamado $\mathrm{Z}$ score, Equação 2, que representa uma avaliação do laboratório. É calculado por:

$Z=\frac{\left(X_{l a b}-X_{r e f}\right)}{s_{r e f}}$

onde $X$ é valor de concentração; $s$ é a unidade de desvio padrão e, $l a b$ e ref são índices relacionados à medição do laboratório e do material de referência usado.

Se $|Z| \leq 2$, satisfatório; $2<|Z| \leq 3$, questionável e $|Z|>3$, insatisfatório.

Os valores determinados para os materiais de referência SRM 679 e 2711, limite de determinação e Z score são apresentados nas Tabelas 1 e 2 . Os valores de $Z$ score dependem do material de referência usado. No caso do material de referência SRM 679, são satisfatórios: $\mathrm{Al}, \mathrm{Cr}, \mathrm{Fe}, \mathrm{K}, \mathrm{Sr}$ e Ti, porém, questionável para Si. No caso de SRM 2711, são satisfatórios: Ba, Ca, Fe, K, Mn, S, Ti, $\mathrm{V}$ e $\mathrm{Zn}$, entretanto, é questionável para $\mathrm{Pb}$ e $\mathrm{S}$. O valor apresentado para $\mathrm{Rb}$ é apenas indicativo. Esses elementos foram utilizados porque os desvios padrões relativos são $\leq 10 \%$ e a exatidão $\leq 8 \%$. Foram utilizados dois materiais de referência porque o material certificado de argila, Brick Clay, não apresenta valores certificados para todos os elementos utilizados neste trabalho.

\section{Homogeneidade dos resultados obtidos por EDXRF}

A homogeneidade da amostra e dos materiais de referência pode ser verificada na Figura 2. Este gráfico foi obtido aplicando-se a análise de componentes principais após a transformação de variáveis para logaritmo da concentração dos elementos $\mathrm{Al}, \mathrm{Ba}, \mathrm{Ca}, \mathrm{Cr}$, Fe, K, Mn, Pb, Rb, S, Si, Sr, Ti, V, Zn.

Esse gráfico mostra que não há uma dispersão muito grande dos valores de concentração entre as amostras de mesma espécie. Mostra a formação de grupos para uma amostra de cerâmica (18) e para os materiais de referência, SRM 679, SRM 2709, SRM 2710 e SRM 2711 indicando homogeneidade do conjunto analisado. As elipses apresentam os agrupamentos separados com 95\% de confiança.

Para verificar a influência da quantidade de material, foram analisadas pastilhas com 1,$50 ; 2,00$ e 5,00 g e pressão de

Tabela 1. Valores obtidos para o material de referência Brick Clay - SRM 679

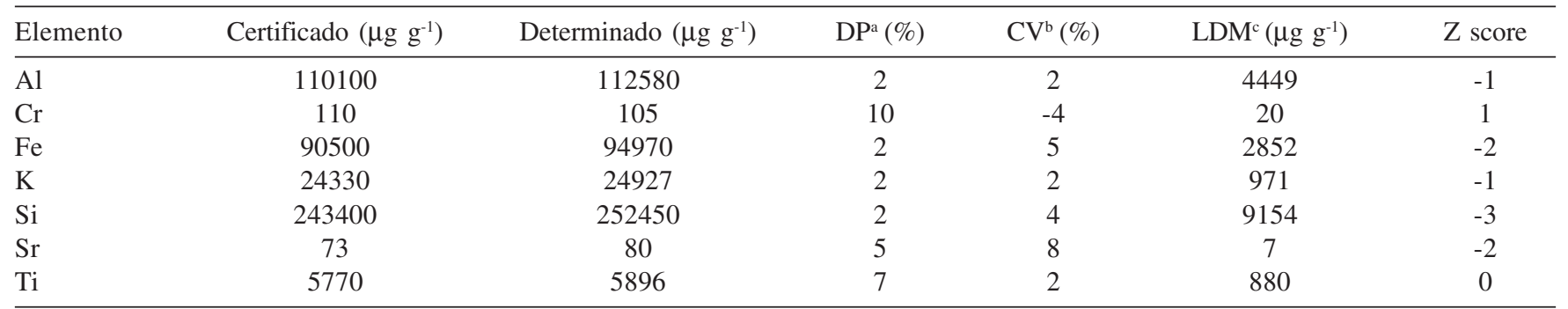

a) desvio padrão relativo; b) coeficiente de variação; c) limite de determinação do método 
Tabela 2. Valores obtidos para o material de referência Montana Soil - SRM 2711

\begin{tabular}{|c|c|c|c|c|c|c|}
\hline Elemento & Certificado $\left(\mu \mathrm{g} \mathrm{g}^{-1}\right)$ & Determinado $\left(\mu \mathrm{g} \mathrm{g}^{-1}\right)$ & $\mathrm{DP}^{\mathrm{a}}(\%)$ & $\mathrm{CV}^{\mathrm{b}}(\%)$ & $\mathrm{LDM}^{\mathrm{c}}\left(\mu \mathrm{g} \mathrm{g}^{-1}\right)$ & Z score \\
\hline $\mathrm{Ba}$ & 726 & 698 & 4 & -4 & 58 & 1 \\
\hline $\mathrm{Ca}$ & 28800 & 28285 & 5 & -2 & 3109 & 1 \\
\hline $\mathrm{Fe}$ & 28900 & 29368 & 5 & 2 & 2948 & -1 \\
\hline $\mathrm{K}$ & 24500 & 22817 & 6 & -7 & 2522 & 2 \\
\hline Mn & 638 & 682 & 5 & 7 & 68 & -2 \\
\hline $\mathrm{Pb}$ & 1162 & 1080 & 5 & -7 & 102 & 3 \\
\hline $\mathrm{Rb}^{\mathrm{d}}$ & 110 & 109 & 4 & -1 & 8 & \\
\hline$S$ & 420 & 450 & 3 & 7 & 25 & -3 \\
\hline $\mathrm{Ti}$ & 3060 & 2852 & 1 & -7 & 77 & 1 \\
\hline V & 81,6 & 81 & 6 & -1 & 10 & 0 \\
\hline $\mathrm{Zn}$ & 350,4 & 353 & 5 & 1 & 37 & -1 \\
\hline
\end{tabular}

a) desvio padrão relativo; b) coeficiente de variação; c) limite de determinação do método; d) valor indicativo

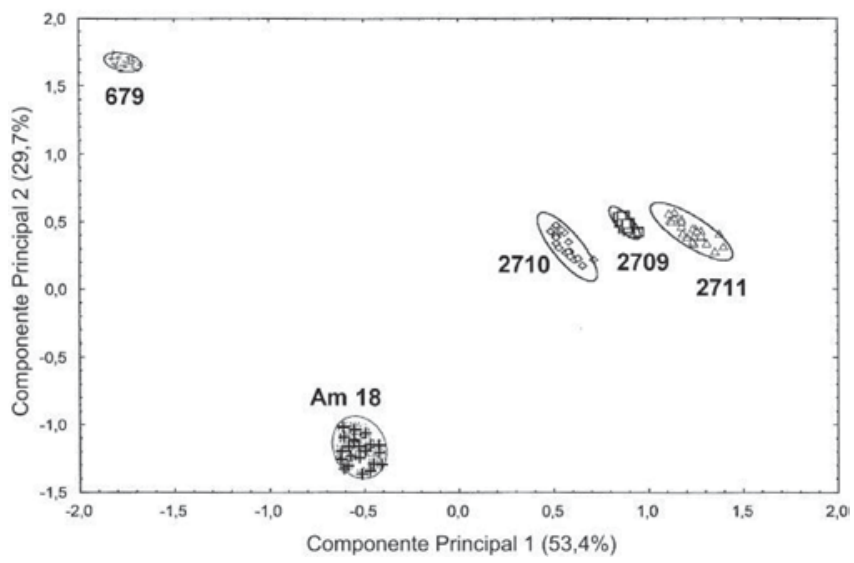

Figura 2. Gráfico de componentes principais para verificação da homogeneidade da amostra de cerâmica e dos materiais de referência SRM 679, 2709, 2710 e 2711. Elipses de 95\% de confiabilidade

compactação constante de 10 t. O gráfico de componentes principais da Figura 3 representa a distribuição das varreduras obtidas para estas pastilhas. Verifica-se que não há formação de grupos. Ou seja, não há uma influência marcante da massa da pastilha na concentração dos elementos analisados.

Em relação à pressão de compactação, foram analisadas pastilhas com pressão de compactação de 5 e 10 t e massa de 2,00 g. O gráfico de componentes principais da Figura 4 mostra que a variação na pressão de compactação provoca alterações na concentra-

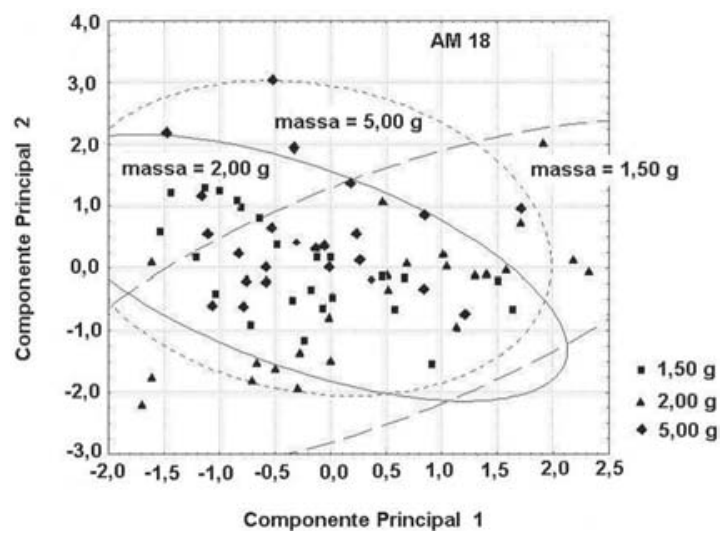

Figura 3. Gráfico de componentes principais para verificação da dependência com a massa da pastilha. Análise por EDXRF de pastilhas de massas 1,50; 2,00 e 5,00 g e mesma pressão de compactação. Elipses de $95 \%$ de confiabilidade ção dos elementos analisados. Portanto, uma vez escolhida, a pressão de compactação deve ser mantida para todo o ensaio.

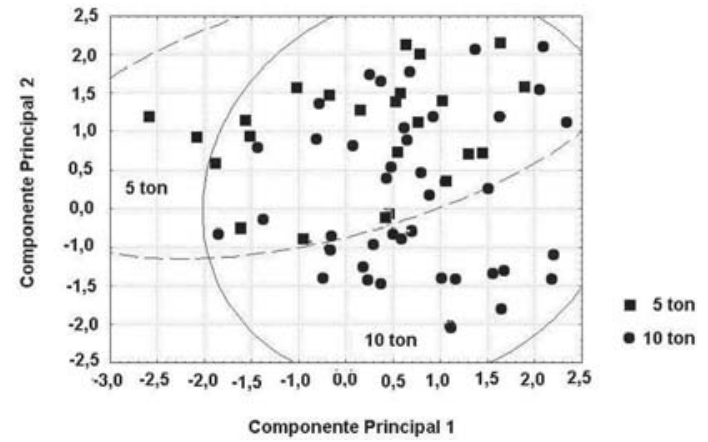

Figura 4. Gráfico de componentes principais para verificação da dependência com a pressão de compactação da pastilha. Análise por EDXRF de pastilhas de mesma massa, mas com pressão de compactação de 5 e $10 t$. Elipses de 95\% de confiabilidade

\section{Análise de amostras de cerâmica arqueológica por EDXRF}

Foram analisadas 120 amostras de 7 sítios, distribuídas conforme indicado na Tabela 3.

O intervalo de variação da concentração das amostras de cerâmica analisadas é apresentado na Tabela 4. A estes dados foi aplicada a análise de componentes principais, após a transformação de variável para logaritmo da concentração.

A partir da análise de componentes principais construiu-se o gráfico da Figura 5. Verifica-se nesta figura a existência de 3 grupos e interações entre as amostras. As elipses apresentam os agrupamentos

Tabela 3. Número de amostras analisadas por sítio, sua localização em relação ao rio e a distância relativa ao sítio Wilmoth Röpke

\begin{tabular}{lccc}
\hline Sítio & Localidade & $\begin{array}{c}\text { Distância } \\
(\mathrm{km})^{\mathrm{a}}\end{array}$ & $\begin{array}{c}\text { Amostras } \\
\text { analisadas }\end{array}$ \\
\hline Nilton Puntel & Alto Jacuí & 11 & 14 \\
Gentil Menegasse & Alto Jacuí & 5 & 16 \\
Wilmoth Röpke & Médio Jacuí & 0 & 27 \\
Marsílio Bartz & Médio Jacuí & 5 & 26 \\
Cabeceira do Raimundo & Vacacaí Mirim & 69 & 12 \\
Pedra Grande & Ibicuí Mirim & 95 & 9 \\
São Lourenço Mártir & Ijuí & 182 & 16 \\
Total de amostras analisadas & & & 120 \\
\hline
\end{tabular}

a) Em linha reta em relação ao sítio Wilmoth Röpke 
Tabela 4. Intervalo de variação da concentração dos elementos químicos presentes nas amostras dos sítios analisados

\begin{tabular}{lcc}
\hline Elemento & $\begin{array}{c}\text { Concentração } \\
\text { máxima }\left(\mu \mathrm{g} \mathrm{g}^{-1}\right)\end{array}$ & $\begin{array}{c}\text { Concentração } \\
\text { mínima }\left(\mu \mathrm{g} \mathrm{g}^{-1}\right)\end{array}$ \\
\hline $\mathrm{Al}$ & 93910 & 40070 \\
$\mathrm{Ba}$ & 8436 & 317 \\
$\mathrm{Ca}$ & 40010 & 240 \\
$\mathrm{Cr}$ & 166 & 24 \\
$\mathrm{Fe}$ & 96410 & 9520 \\
$\mathrm{~K}$ & 22920 & 2630 \\
$\mathrm{Mn}$ & 6280 & 140 \\
$\mathrm{~Pb}$ & 202 & 30 \\
$\mathrm{Rb}$ & 185 & 28 \\
$\mathrm{~S}$ & 370 & 47 \\
$\mathrm{Si}$ & 297720 & 130880 \\
$\mathrm{Sr}$ & 172 & 8 \\
$\mathrm{Ti}$ & 32687 & 1250 \\
$\mathrm{~V}$ & 895 & 41 \\
$\mathrm{Zn}$ & 272 & 26 \\
\hline
\end{tabular}

separados com $95 \%$ de confiança. Os dados representados por retângulos e círculos cheios foram descartados, considerados como amostras intrusivas ("outliers"). A detecção dos "outliers" foi feita via componente principal e esse método é descrito por Ribeiro ${ }^{24}$. Os três agrupamentos correspondem às amostras do sítio São Lourenço Mártir (rio Ijuí), dos sítios Pedra Grande e Cabeceira do Raimundo (Rios Ibicuí Mirim e Vacacaí Mirim) e dos sítios Wilmoth Röpke, Gentil Menegasse, Nilton Puntel e Marsílio Bartz (Rio Jacuí).

$\mathrm{O}$ gráfico mostra interações entre as amostras dos sítios dos rios Jacuí, Vacacaí Mirim e Ibicuí Mirim, mas não desses grupos com as do sítio São Lourenço Mártir. Esse fato pode ser explicado lembrando-se que o sítio São Lourenço Mártir é considerado um sítio histórico (pós-contato com os europeus) e os outros são considerados como pré-contato (antes do contato com os europeus) e, portanto, todos os sítios estudados podem não ser contemporâneos.

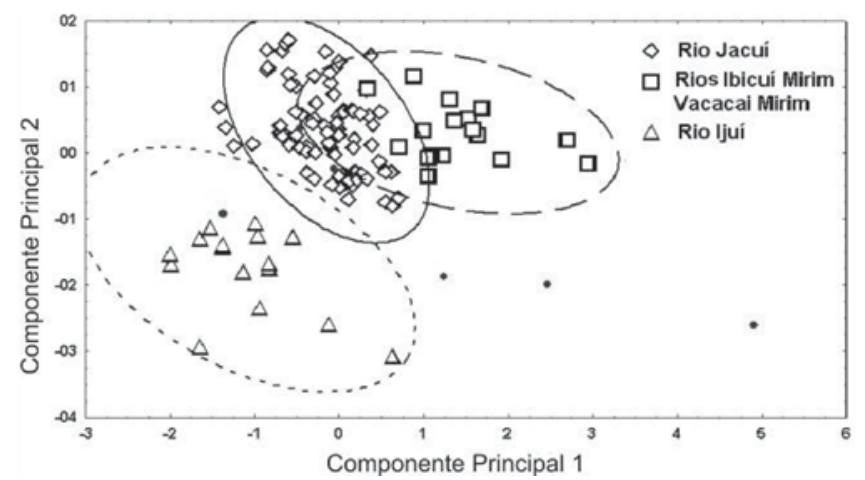

Figura 5. Gráfico de componentes principais para as amostras analisadas dos sítios arqueológicos do Rio Grande do Sul. Elipses de 95\% de confiabilidade

Os sítios Cabeceira do Raimundo e Pedra Grande distam entre si $25,5 \mathrm{~km}$. A análise de componentes principais para as amostras destes sítios, assim como o gráfico do logaritmo da concentração de $\mathrm{Cr}$ e Ti (Figura 6) mostrou que podem ter havido interações entre os habitantes destes sítios. Outra possibilidade é que a matéria-prima possa ter origem em lugares próximos, de modo que a composição química da cerâmica seja semelhante.

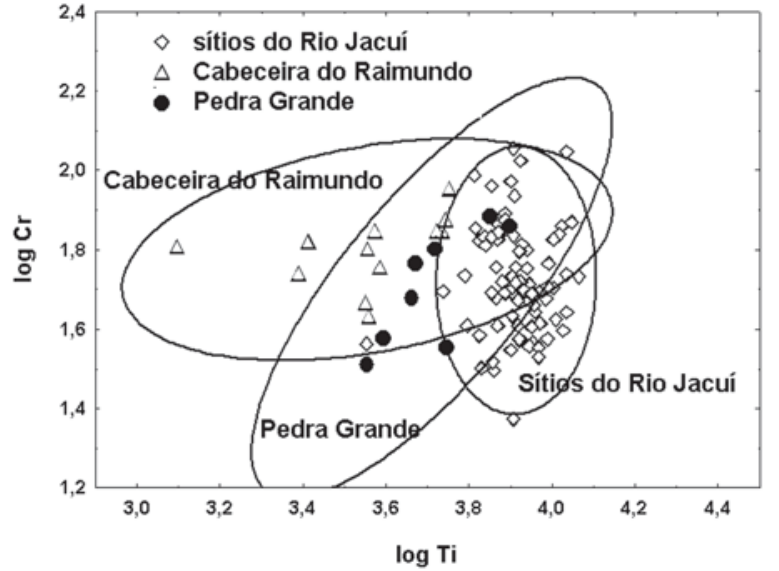

Figura 6. Gráfico do logaritmo da concentração de Cr e Ti para amostras dos sítios Cabeceira do Raimundo, Pedra Grande e alguns sítios do Vale do Jacuí, no em torno dos Rios Vacacaí Mirim, Ibicuí Mirim e Vale do Rio Jacuí. Elipses de $95 \%$ de confiabilidade

Verifica-se que as amostras dos sítios Pedra Grande e Cabeceira de Raimundo, estão separadas das amostras dos sítios do Rio Jacuí. A distância entre estes sítios é variada, mas é maior que 70 km. O Rio Ibicuí Mirim é afluente do Rio Uruguai, enquanto que o Rio Vacacaí Mirim é afluente do Rio Jacuí que deságua na Lagoa dos Patos; entretanto, nesta região existem vários arroios e riachos que poderiam ser usados como ligação entre estes sítios. Esse fato pode ser verificado na Figura 1.

O mapa Etnográfico Histórico do Rio Grande do Sul de Carlos Teschauer mostra que esta região do Rio Ibicuí Mirim fazia parte do território dos Guaranis, enquanto que a região do Rio Jacuí poderia ter sido território dos Tapes ${ }^{2}$. Embora ambos fossem de cultura Guarani, os colonizadores europeus registraram denominações diferentes mas isso não invalida a tese de que poderiam ser grupos distintos. A interação entre as amostras destas regiões, verificada na análise de componente principal, pode significar a interação entre estes dois grupos, pois a cerâmica produzida era semelhante na decoração.

As interações entre os sítios do Rio Jacuí parecem ter sido mais intensas que as dos Rios Ibicuí Mirim e Vacacaí Mirim e as do Rio Jacuí (Figura 7). A região do Rio Jacuí foi densamente povoada conforme atestam os inúmeros sítios arqueológicos encontrados 4,24,26-28. Na região do Baixo Rio Jacuí, há também registros de várias reduções jesuíticas ${ }^{2,3}$. Na Figura 7 são apresentadas as elipses de $95 \%$ de confiabilidade para a separação das amostras de Gentil

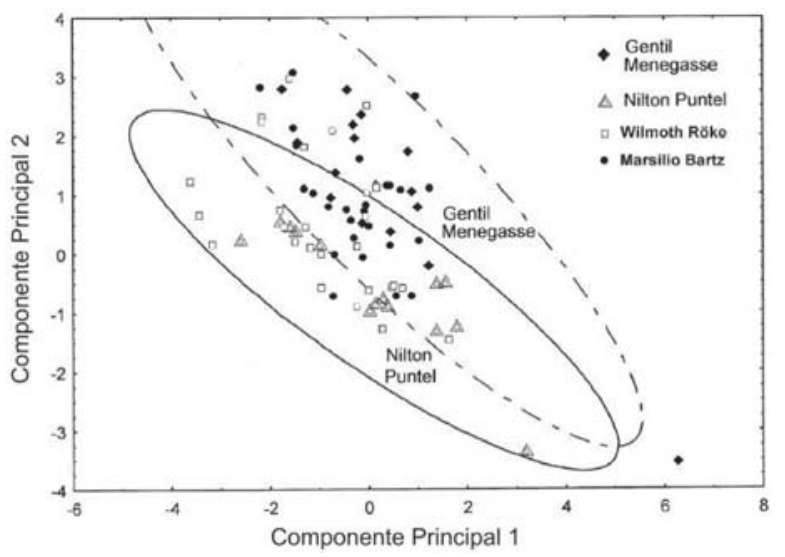

Figura 7. Gráfico de componentes principais para os sítios da região do Rio Jacuí. Elipse de confiabilidade de 95\% para os sítios Nilton Puntel e Gentil Menegasse 


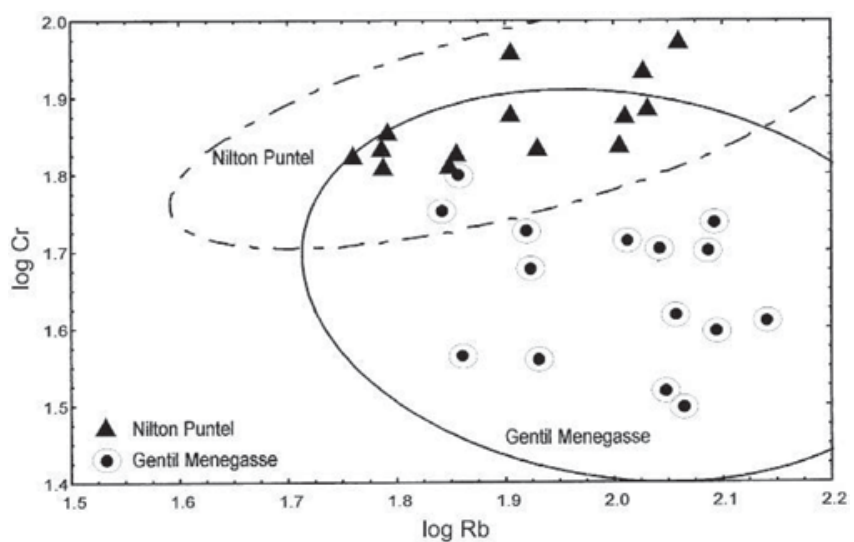

Figura 8. Gráfico de logaritmo da concentração de Rb e Cr, para as amostras dos sítios: Nilton Puntel e Gentil Menegasse (Alto Jacuí). Elipses de 95\% de confiabilidade

\section{Menegasse e Nilton Puntel.}

As amostras dos sítios Nilton Puntel e Gentil Menegasse do Alto Jacuí distam entre si $6 \mathrm{~km}$ em linha reta. Apresentam assinaturas químicas, semelhantes à observada no gráfico de componentes principais, que podem ser verificadas quando as amostras dos dois sítios foram apresentadas no gráfico do logaritmo da concentração de $\mathrm{Cr}$ e $\mathrm{Rb}$ (Figura 8).

\section{CONCLUSÃO}

Neste trabalho apresentaram-se os primeiros dados experimentais relativos à análise arqueométrica de cerâmica da região central do Rio Grande do Sul com o uso da técnica de fluorescência de raios $\mathrm{X}$ por dispersão de energia, além da homogeneidade da amostra cerâmica e resultados da análise de componentes principais.

Foram analisadas 120 amostras de 7 sítios, 3 materiais de referência de solo, San Joaquim soil - SRM-2709, Montana soil - SRM2710, Montana soil - SRM-2711 e um de cerâmica, Brick Clay SRM 679.

Para as amostras de cerâmica, verificou-se que com análise multivariada foi possível observar assinaturas químicas de sítios que estão localizados no em torno dos Rios Ijuí, Ibicuí Mirim, Vacacaí Mirim e Jacuí.

As amostras do sítio São Lourenço Mártir mostram ter composição química diferente das amostras dos outros sítios, pois se apresentaram como um grupo separado.

Sítios como Cabeceira de Raimundo e Pedra Grande, distantes entre si aproximadamente $25,5 \mathrm{~km}$, apresentaram uma discreta separação. Algumas amostras se encontram sobrepostas. Estas amostras podem ser devidas a algum tipo de interação social ou uso de matéria-prima de origem semelhante.

As amostras dos sítios do Rio Jacuí apresentaram sobreposição, demonstrando que podem ter sido fabricadas com matéria-prima de mesma localidade ou ter havido interações de troca, como demonstra a grande quantidade de sítios escavados na região. Amostras do sítio Nilton Puntel, localizado a $6 \mathrm{~km}$ em linha reta do sítio Gentil Menegasse, apresentam pequena sobreposição de amostras, mostrando que as interações se houve, foram pequenas.
O método utilizado pode ser usado para verificar assinaturas químicas entre amostras de sítios que apresentam distâncias na ordem de dezenas de quilômetros. Também é possível, a partir de interações entre amostras, apresentar indícios sobre relações entre sítios, consistente com dados arqueológicos, etnográficos e históricos.

\section{AGRADECIMENTOS}

À Shimadzu do Brasil pelo uso do espectrômetro de fluorescência de raios X por dispersão de energia, EDX-900.

\section{REFERÊNCIAS}

1. Brochado, J. P.; Clio 1991, 4, 85.

2. Soares, A. L. R.; Guarani: Organização Social e Arqueologia, EDIPUCRS: Porto Alegre, 1997.

3. Soares, A. L. R. Em Rio Grande do Sul: Quatro Séculos de História; Quevedo, J., org.; Martins Livreiro: Porto Alegre, 1999.

4. Rogge, J. H.; Arqueologia do Rio Grande do Sul, Brasil, Documento 06 , UNISINOS: São Leopoldo, 1996.

5. http://www.trabalhoindigenista.org.br/Docs/artigo_ISA_Adriana_Felipim.pdf, acessada em Outubro 2005.

6. Noelli, F. S.; Revista da USP 1999-2000, 44, 218.

7. Fajardo, F.; Dissertação de Mestrado, Universidade Federal de Santa Maria, Brasil, 2001.

8. Soares, A. L. R.; Tese de Doutorado, Universidade de São Paulo, Brasil, 2004.

9. Ribeiro P. A. M. Em Escritos sobre Arqueologia; Ribeiro, P. A. M.; Nunes, C. O. I., eds.; Fundação Universidade Federal do Rio Grande: Porto Alegre, 2001.

10. Marengo, E.; Aceto, M.; Robotti, E.; Liparota, M. C.; Bobba, M.; Pantó, G.; Anal. Chim. Acta 2005, 537, 359.

11. Tsolakidou, A.; Kilikoglou, V.; Anal. Bioanal. Chem. 2002, 374, 566.

12. Munita, C. S.; Silva, M. A.; Silva, F. A.; Oliveira, P. M. S.; Instrum. Sci. Technol. 2005, 33, 161.

13. Appoloni, C. R.; Espinoza Quiñones, F. R.; Aragão, P. H. A.; Santos, A. O. dos; Silva, L. M. da; Barbieri, P. F.; Nascimento Filho, V. F. do; Coimbra, M. M.; Radiat. Phys. Chem. 2001, 61, 711.

14. Felicíssimo, M. P.; Peixoto, J. L. S.; Tomasi, R.; Azioune A.; Pireaux, J. J.; Houssiau, L.; Rodrigues, U. P.; Philos. Mag. 2004, 84, 3483.

15. Felicíssimo, M. P.; Peixoto, J. L. S.; Barbosa, M. S.; Demortier, G.; Pireaux, J. J.; Rodrigues Filho, U. P.; J. Radioanal. Nucl. Chem. 2006, 267, 209.

16. Adan-Bayewitz, D.; Archaeometry 1999, 41, 1.

17. Leung, P. L.; Stokes, M. J.; Li, M. T. W.; Peng, Z. C.; Wu, S. C.; X-Ray Spectrom, 1998, 271, 11.

18. Druc, I. C.; Gwyn, Q. H. J.; J. Archaeol. Sci. 1998, 25, 707.

19. http://www.missoes.iphan.gov.br/outrosp/piv.htm, acessada em Abril 2006.

20. Silva, V. H. O. da; Prade, E. E.; Kelin, A. I.; Biblos. 1992, 4, 43.

21. Schwedt, A.; Hommsen, H.; Zacharias, N.; Archaeometry 2004, 46, 85.

22. Rousseau, R. M.; The Rigaku J. 2001, 18, 33.

23. Instituto Nacional de Metrologia, Normalização e Qualidade Industrial (INMETRO); Orientações sobre Validações de Métodos de Ensaios Químicos, DOQ-CGCRE-008, 2003, rev. 1.

24. Ribeiro, F. A. de L.; Dissertação de Mestrado, Universidade Estadual de Campinas, Brasil, 2001.

25. Dias, A. S.; Tese de Doutorado, Universidade de São Paulo, Brasil, 2003.

26. Schmitz, P. I.; Becker, I. B.; Ribeiro, P. A. M.; Baumhardt, G.; Baumhardt, U.; Martin, H.; Steinhaus, R.; Brochado, J. P.; Arqueologia do Vale do Rio Pardinho (Comparações com material proveniente do Alto Jacuí) $1^{a}$. Parte, Pesquisas, série Antropologia, 23, Instituto Anchietano de Pesquisas: São Leopoldo, 1970.

27. Klamt, S. C.; Uma Contribuição para o Sistema de Assentamento de um Grupo Horticultor da Tradição Cerâmica Tupiguarani, Série Conhecimento, 29, EDUNISC: Santa Cruz do Sul, 2005.

28. Schmitz, P. I.; Rogge, J. H.; Arnt, F. V.; Arqueologia do Rio Grande do Sul, Brasil, Documento 08, UNISINOS: São Leopoldo, 2000. 